\title{
Nowhere-zero 3-flows in Cayley graphs and Sylow 2-subgroups
}

\author{
Mária Nánásiová • Martin Škoviera
}

Received: 26 October 2007 / Accepted: 12 September 2008 / Published online: 26 September 2008 (C) Springer Science+Business Media, LLC 2008

\begin{abstract}
Tutte's 3-Flow Conjecture suggests that every bridgeless graph with no 3edge-cut can have its edges directed and labelled by the numbers 1 or 2 in such a way that at each vertex the sum of incoming values equals the sum of outgoing values. In this paper we show that Tutte's 3-Flow Conjecture is true for Cayley graphs of groups whose Sylow 2-subgroup is a direct factor of the group; in particular, it is true for Cayley graphs of nilpotent groups. This improves a recent result of Potočnik et al. (Discrete Math. 297:119-127, 2005) concerning nowhere-zero 3-flows in abelian Cayley graphs.
\end{abstract}

Keywords Nowhere-zero flow · Cayley graph · Group centre · Sylow subgroup · Nilpotent group

\section{Introduction}

In 1972 Tutte proposed the following conjecture (see [1, unsolved problem 48]) which complements his two earlier conjectures on nowhere-zero flows on graphs, the 5-Flow Conjecture [16] and the 4-Flow Conjecture [17].

Conjecture (3-Flow Conjecture) Every bridgeless graph with no 3-edge-cut has a nowhere-zero 3-flow.

\footnotetext{
M. Nánásiová · M. Škoviera (凶)

Department of Computer Science, Faculty of Mathematics, Physics and Informatics, Comenius University, 84248 Bratislava, Slovakia

e-mail: skoviera@dcs.fmph.uniba.sk

M. Nánásiová

e-mail: meri@ksp.sk
} 
The motivation for the conjecture came from Grötzsch's theorem [5] stating that every triangle-free planar graph is 3-colourable. By the plane duality, every bridgeless planar graph with no 3-edge-cut has a nowhere-zero 3-flow. The 3-Flow Conjecture thus extends the validity of the dual Grötzsch Theorem to arbitrary graphs.

The present status of the 3-Flow Conjecture can be briefly summarised as follows. The conjecture has been verified for a number of infinite classes of graphs, including the projective-planar graphs [14], Cartesian product graphs [6, 13], locally connected graphs $[2,10]$, and the squares of graphs [18]. Furthermore, the conjecture can be reduced to 5-edge-connected 5-regular graphs $[8,9,20]$, and is true for random graphs [15].

As far as flows on highly symmetrical graphs are concerned, it is known that every vertex-transitive graph of valency greater than three has a nowhere-zero 4-flow. This is an easy consequence of the 4-Flow Theorem of Jaeger about flows in 4-edgeconnected graphs (see, for example, Jaeger [7, Theorem 4.7]) combined with the fact that every $k$-valent vertex-transitive graph is $k$-edge-connected. By the 3 -Flow Conjecture, however, every vertex-transitive graph of valency at least four should support a nowhere-zero 3-flow. It is therefore tempting to examine the existence of nowhere-zero 3-flows on as many vertex-transitive graphs as possible.

Among vertex-transitive graphs, Cayley graphs are best understood, and therefore are usually the first to be investigated. In spite of that, only Cayley graphs of abelian groups are so far known to have nowhere-zero 3-flows (Potočnik et al. [11]). The purpose of the present paper is to extend the result of [11] to a much wider class of Cayley graphs.

Before stating our main result we make the observation that one only needs to consider Cayley graphs of odd valency greater than four and groups of even order (see the last paragraph of Sect. 2 for details). In particular, the set of generators for such a Cayley graph necessarily includes an involution. We show that if an involution in the generating set belongs to the group centre, then the graph admits a nowherezero 3-flow (Theorem 3.3). A combination of this theorem with graph coverings and induction leads to the following two results.

Theorem Let $G$ be a finite group in which a Sylow 2-subgroup constitutes a direct factor of the group. Then every Cayley graph $\mathrm{Cay}(G, S)$ of valency at least four has a nowhere-zero 3-flow.

Corollary Every Cayley graph of valency at least four on a nilpotent group has a nowhere-zero 3-flow.

\section{Preliminaries}

\subsection{Graphs}

All graphs in this paper will be finite, with multiple edges and loops permitted. We describe graphs as quadruples $K=(D, V, I, L)$ where $D=D(K)$ is a finite set of darts (directed edges), $V=V(K)$ is a finite set of vertices, $I: D \rightarrow V$ is an incidence 
function which with every dart associates its initial vertex, and $L$ is an involution of $D$ which to every dart $z$ assigns its inverse dart $z^{-1}$. Each edge of $K$ is formed by a pair of mutually inverse darts. In other words, we may think of the dart-set of a graph $K$ as being obtained from its edge-set by replacing each edge of $K$ (even a loop) with a pair of darts that are incident with the same vertices but have opposite direction. For an arbitrary vertex $v$, we let $D(v)$ be the set of all darts emanating from $v$. The cardinality of $D(v)$ is the valency of $v$.

Let $A$ by an arbitrary abelian group, written additively. We define an $A$-flow on $K$ as a function $\xi: D(K) \rightarrow A$ satisfying the following two conditions:

(F1) $\xi\left(z^{-1}\right)=-\xi(z)$, for each dart $z \in D(K)$,

(F2) $\sum_{z \in D(v)} \xi(z)=0$, for each vertex $v \in V(K)$.

A flow $\xi$ is said to be nowhere-zero if $\xi(z) \neq 0$ for each dart $z \in D(K)$. A nowherezero $k$-flow is a $\mathbb{Z}$-flow which takes values in the set $\{ \pm 1, \ldots \pm(k-1)\}$. Clearly, a graph which has a nowhere-zero $k$-flow also has a nowhere-zero $(k+1)$-flow.

Note that for any two $A$-flows $\xi$ and $\tau$ on a graph $K$ the functions $-\xi$ and $\xi+\tau$ are again $A$-flows. Nevertheless, the sum of nowhere-zero flows need not be nowherezero.

It is often convenient to describe a flow on a graph as a sum of flows on subgraphs. In doing that, we will automatically view each flow on a subgraph as a flow defined on the whole graph but with zero values outside the subgraph.

For further information on nowhere-zero flows the reader may consult Diestel [3, Chapter 6], Jaeger [7], or Zhang [19].

\subsection{Groups}

All groups considered in this paper will be finite, with the unique exception of the group of integers $\mathbb{Z}$. A group $G$ is a direct product of subgroups $H$ and $K$ provided that both $H$ and $K$ are normal in $G$, the product $H K$ is the whole of $G$, and $H \cap K=1$. If this is the case, then each of $H$ and $K$ is a direct factor of $G$. The centre of a group $G$, denoted by $Z(G)$, is the set of all elements of $G$ that commute with every element of $G$. Elements that belong to $Z(G)$ are called central. It is well known that $Z(G)$ is a characteristic subgroup of $G$, one which is invariant under all automorphisms of $G$, and that every subgroup of the centre is a normal subgroup of the whole group.

A group $G$ is called a $p$-group for a prime $p$ if each element of $G$ has order some power of $p$. It is well known that the order of each finite $p$-group is a power of $p$, and vice versa. Furthermore, every finite $p$-group has a non-trivial centre; in fact, it contains a central element of order $p$.

Assume that $G$ has order $p^{m} n$ where $n$ is not divisible by $p$. Then $G$ always contains a subgroup of order $p^{m}$, called a Sylow $p$-subgroup. It is well known that each $p$-subgroup of $G$ is contained in some Sylow $p$-subgroup and that any two Sylow $p$-subgroups are conjugate in $G$.

A group $G$ is said to be nilpotent if it contains a normal series $G=G_{0} \unrhd$ $G_{1} \unrhd \cdots \unrhd G_{s}=1$ of normal subgroups such that each $G_{i} / G_{i+1}$ is contained in $Z\left(G / G_{i+1}\right)$. Equivalently, a group is nilpotent if and only if starting from $G$ one can 
reach the trivial group by a repeated factorisation by the centre. It is well known that a subgroup and a homomorphic image of each nilpotent group are again nilpotent. Moreover, every finite nilpotent group is a direct product of its Sylow subgroups. In particular, every finite $p$-group is nilpotent.

More details on the presented group theory material can be found in any standard group theory textbook, see for example, [12].

\subsection{Cayley graphs}

Let $G$ be a group and let $S=\left(s_{1}, s_{2}, \ldots, s_{n}\right)$ be a sequence of elements of $G-\{1\}$ such that the mapping $s_{i} \mapsto s_{i}^{-1}$ permutes the entries of $S$. We call $S$ a connection sequence for $G$. (Note that we allow connection sequences to contain repeated elements.) Define the Cayley graph $\operatorname{Cay}(G, S)$ of $G$ with connection sequence $S$ by taking the vertices of $\operatorname{Cay}(G, S)$ to be the elements of $G$ and the darts of $\operatorname{Cay}(G, S)$ to be the ordered pairs $\left(g, s_{i}\right)$ where $g \in G$ and $s_{i} \in S$. An arbitrary dart $\left(g, s_{i}\right)$ will have initial vertex $g$ and terminal vertex $g s_{i}$; its inverse is defined by setting $\left(g, s_{i}\right)^{-1}=\left(g s_{i}, s_{i}^{-1}\right)$. Thus the Cayley graph $\operatorname{Cay}(G, S)$ is regular of valency $|S|$.

Note that a Cayley graph is connected if and only if the elements of the connection sequence generate the whole Cayley group $G$. Although it is natural to concentrate on connected Cayley graphs, it is often convenient to deal with Cayley subgraphs that need not be connected. Therefore we allow our Cayley graphs to be disconnected.

If a Cayley graph $\operatorname{Cay}(G, S)$ has odd valency, then $S$ must contain an involution. It follows that if $G$ has odd order, then any Cayley graph of $G$ has even valency. Since every graph with all vertices of even valency has a nowhere-zero 2-flow ([3, Proposition 6.4.1]), every Cayley graph of a group of odd order has a nowhere-zero 2-flow. Thus in the study of nowhere-zero 3-flows on Cayley graphs it is sufficient to restrict to Cayley graphs $\operatorname{Cay}(G, S)$ where $G$ has even order and $S$ contains an involution.

\section{Cayley graphs with central involutions}

In this section we show that every Cayley graph of valency at least four whose connection sequence contains a central involution admits a nowhere-zero 3-flow. Before proving this result we need to look at 3-flows on certain cubic subgraphs called closed ladders.

Let $L_{n}$ denote the Cartesian product $P_{n-1} \square K_{2}$ of the path $P_{n-1}$ of length $n-1$ with the complete graph $K_{2}$ of order two (for the definition of the Cartesian product of graph we refer the reader to [1, p. 96]). Assume that $V\left(P_{n-1}\right)=\{0,1, \ldots, n-1\}$ and $V\left(K_{2}\right)=\{0,1\}$. By adding the edges $(n-1,0)(0,0)$ and $(n-1,1)(0,1)$ to $L_{n}$ we obtain the graph $C_{n} \square K_{2}$ where $C_{n}$ is the circuit of length $n$. We call the latter graph the circular ladder and denote it by $C L_{n}$. If the edges $(n-1,0)(0,1)$ and $(n-1,1)(0,0)$ are added to $L_{n}$, the Moebius ladder $M L_{n}$ is obtained. For $n=1$ these definitions imply that $C L_{1}$ has two loops joined by a bridge while $M L_{1}$ has three parallel edges. Any graph isomorphic to either $C L_{n}$ or $M L_{n}$ for some $n$ will be referred to as a closed ladder. 
The following proposition explains how closed ladders are related to central involutions. Before stating the result we only recall that $\mathbb{D}_{n}$ denotes the dihedral group of order $2 n$ generated by two elements $s$ and $t$ satisfying the relations $s^{n}=1, t^{2}=1$, and $t s t=s^{-1}$.

Proposition 3.1 Let $\operatorname{Cay}(G, S)$ be a connected cubic Cayley graph such that $S$ contains a central involution of $G$. Then $G$ is isomorphic to one of following groups: $\mathbb{Z}_{2 n}$, $\mathbb{Z}_{n} \times \mathbb{Z}_{2}, \mathbb{D}_{2 n}$ or $\mathbb{D}_{n} \times \mathbb{Z}_{2}$. In each case, $\operatorname{Cay}(G, S)$ is a closed ladder.

Proof Since Cay $(G, S)$ is a cubic graph, $S$ contains either one or three involutions, one of which is an involution $c \in Z(G)$. If $S$ consists of three involutions, say, $x, y$, and $c$, then either $G \cong \mathbb{D}_{2 n}$ or $G=\mathbb{D}_{n} \times \mathbb{Z}_{2}$ depending on whether $c$ does or does not belong to $\langle x, y\rangle$. If $S=\left(x, x^{-1}, c\right)$ where $x$ is an element of order greater than 2 , then $G \cong \mathbb{Z}_{2 n}$ provided that $c \in\langle x\rangle$, or $G \cong \mathbb{Z}_{n} \times \mathbb{Z}_{2}$ otherwise. The reader can readily verify that in all these cases $\operatorname{Cay}(G, S)$ is a closed ladder.

It is well known that a cubic graph has a nowhere-zero 3-flow if and only if it is bipartite (see, for example, Diestel [3, Proposition 6.4.2]). Furthermore, it is clear from the definition that the circular ladder $C L_{n}$ is bipartite if and only if $n$ is even while the Moebius ladder $M L_{n}$ is bipartite if and only if $n$ is odd. By combining these two facts we obtain the following lemma.

Lemma 3.2 Every closed ladder has a nowhere zero 3-flow or has a 3-flow where the zero value occurs on an arbitrary single rung.

Proof It is sufficient to realise that any circular ladder $C L_{n}$ with one rung removed is homeomorphic to $C L_{n-1}$, and similarly, any Moebius ladder $M L_{n}$ with one rung removed is homeomorphic to $M L_{n-1}$.

Now we are ready for the main result of this section.

Theorem 3.3 Let $\operatorname{Cay}(G, S)$ be a Cayley graph of valency at least four such that $S$ contains a central involution. Then $\operatorname{Cay}(G, S)$ has a nowhere zero 3-flow.

Proof Let $Q=\operatorname{Cay}(G, S)$ and let $c$ be a central involution of $G$ contained in $S$. If the valency of $Q$ is even, then $Q$ obviously admits a nowhere-zero 3-flow. So let the valency of $Q$ be odd. In this case, $S$ contains two connection subsequences $S_{1}$ and $S_{2}$ with $S_{1} \cap S_{2}=\{c\}$ such that the graphs $Q_{1}=\operatorname{Cay}\left(G, S_{1}\right)$ and $Q_{2}=\operatorname{Cay}\left(G, S_{2}\right)$ are cubic. Since the graph $\operatorname{Cay}\left(G, S-\left(S_{1} \cup S_{2}\right)\right)$ has even valency, it is sufficient to find a nowhere-zero 3-flow on the Cayley graph Cay $\left(G, S_{1} \cup S_{2}\right)=Q_{1} \cup Q_{2}$ of valency 5 . Without loss of generality we may assume that $Q=Q_{1} \cup Q_{2}$ and that this graph is connected (in other words, $S_{1} \cup S_{2}$ generates the whole of $G$ ).

From Proposition 3.1 we see that the components of each of $Q_{1}$ and $Q_{2}$ are isomorphic closed ladders. We call them $c$-ladders of $Q$. Since $S_{1} \cap S_{2}=\{c\}$, any $c$-edge of $Q$ belongs to two different $c$-ladders whereas each of the remaining edges belongs to a single $c$-ladder. 
If one of the $c$-ladders, say a component of $Q_{1}$, admits a nowhere-zero 3-flow, then so does $Q_{1}$ and, in fact, so does the whole $Q$, because $Q-E\left(Q_{1}\right)$ is a 2-factor of $Q$. Thus we may assume that none of the $c$-ladders admits a nowhere-zero 3-flow. By Lemma 3.2, however, each of them has a 3-flow where the zero value occurs on a single rung. We use these flows to build up a nowhere-zero 3-flow on $Q$ inductively.

We construct an increasing series $T_{1} \subset T_{2} \subset \cdots \subset T_{i} \subset \cdots$ of subgraphs of $Q$ where each $T_{i}$ is a union of $i$ distinct $c$-ladders, and on each $T_{i}$ we describe a 3-flow $\phi_{i}$ satisfying the following conditions:

(1) At most one edge of $T_{i}$ carries zero under $\phi_{i}$, and if so, then it is a $c$-edge.

(2) Each edge contained in two $c$-ladders of $T_{i}$ carries a non-zero value under $\phi_{i}$.

For $T_{1}$ we take an arbitrary $c$-ladder and define $\phi_{1}$ to be any 3-flow guaranteed by Lemma 3.2. Assume that the subgraph $T_{i}$ and the flow $\phi_{i}$ have already been constructed for some $i \geq 1$. We now define $T_{i+1}$ and $\phi_{i+1}$. There are two cases to consider.

Case 1 The flow $\phi_{i}$ is nowhere-zero on $T_{i}$. If $T_{i}=Q$, then $\phi_{i}$ is the sought nowherezero 3-flow on $Q$, and the proof is finished. Otherwise, take any unused $c$-ladder $L$ and set $T_{i+1}=T_{i} \cup L$. To define $\phi_{i+1}$, remove from $L$ all the rungs with non-zero values under $\phi_{i}$ thereby obtaining a subgraph $L^{\prime}$. If $L^{\prime}$ has no rungs, then it is 2-valent and therefore admits a nowhere-zero 3-flow. If $L^{\prime}$ does retain some rungs, then it is homeomorphic to a closed ladder, and by Lemma 3.2 it admits a 3-flow with at most one zero edge. Denoting by $\rho$ any of these flows and setting $\phi_{i+1}=\phi_{i}+\rho$ we obtain a 3-flow on $T_{i+1}$ which satisfies Conditions (1) and (2) above.

Case 2 There is a unique edge s in $T_{i}$ whose value under $\phi_{i}$ is zero. By Condition (1), $s$ is a $c$-edge, and by Condition (2), there is a $c$-ladder $M$ that contains $s$ and is not completely contained in $T_{i}$. Set $T_{i+1}=T_{i} \cup M$. To define $\phi_{i+1}$, remove from $M$ all the rungs that carry non-zero values under $\phi_{i}$. Since the rung $s$ is not removed, the resulting graph $M^{\prime}$ is homeomorphic to a closed ladder.

If $s$ is the only rung of $M^{\prime}$, then $M^{\prime}$ is homeomorphic to either $M L_{1}$ or $C L_{1}$. In the former case, $M^{\prime}$ has a nowhere-zero 3-flow, say $\sigma$, and we can set $\phi_{i+1}=$ $\phi_{i}+\sigma$. In the latter case, pick any other rung of $M$, say $r$, and consider the subgraph $M^{\prime} \cup r$. Since $M^{\prime} \cup r$ is homeomorphic to $C L_{2}$, it has a nowhere-zero 3-flow, say $\tau$. Clearly, $\tau$ can be chosen in such a way that the values of $\phi+\tau$ on $r$ belong to the set $\{1,-1,2,-2\}$. This allows us to set $\phi_{i+1}=\phi_{i}+\tau$. In either case, $\phi_{i+1}$ is a nowhere-zero 3-flow on $T_{i+1}$, so Conditions (1) and (2) above are fulfilled.

If $M^{\prime}$ has more than one rung, it admits a 3-flow $\psi$ with at most one zero edge such that the values on $s$ are non-zero. By setting $\phi_{i+1}=\phi_{i}+\psi$ we again obtain a 3-flow satisfying Conditions (1) and (2) above.

Since the graph is finite and the sequence $\left(T_{i}\right)$ is strictly increasing, there is an index $m$ such that $T_{m}$ is all of $Q$. Thus the procedure described in Case 1 and Case 2 will terminate, and since any $c$-edge is contained in exactly two $c$-ladders, it will do so with a nowhere-zero 3-flow on $Q$. This completes the proof. 
The main result of Potočnik et al. [11] can now be easily derived from Theorem 3.3.

Theorem 3.4 Every Cayley graph $\operatorname{Cay}(G, S)$ of valency at least four with $G$ abelian has a nowhere-zero 3-flow.

Proof The conclusion is obvious for Cayley graphs of even valency. If the valency is odd, $S$ must contain an involution. As $G$ is abelian, the involution is central, and the result immediately follows from Theorem 3.3.

\section{Main result}

A serious disadvantage of Theorem 3.3 is that its statement depends on the structure of the connection sequence (generating set) rather than on the Cayley group only. The purpose of this section is to overcome this flaw by combining Theorem 3.3 with graph coverings, group factorisation, and induction.

A covering projection of graphs $K$ and $K^{\prime}$ is a graph epimorphism $f: K \rightarrow K^{\prime}$ which for each vertex $u$ of $K$ maps the edges incident with $u$ bijectively onto the edges incident with $f(u)$. In this case, $K$ is a covering graph of $K^{\prime}$, and $K^{\prime}$ is a quotient of $K$. (For more information about coverings the reader is referred to [4, Chapter 2].)

Coverings can be used as an effective tool in many areas of graph theory, including the study of flows on graphs. In particular, if a quotient $K^{\prime}$ of a graph $K$ is endowed with a nowhere-zero $A$-flow $\phi$, then $\phi$ can be lifted via the covering projection $f$ to a nowhere-zero $A$-flow $\tilde{\phi}$ on $K$. Indeed, it is sufficient to set $\tilde{\phi}(x)=\phi(f(x))$ for any dart $x$ of $K$. Obviously, if $\phi$ is a nowhere-zero $k$-flow, so will be the lifted flow $\tilde{\phi}$.

Assume now that $K=\operatorname{Cay}(G, S)$ is a Cayley graph, $H$ is a normal subgroup of $G$, and that $q: G \rightarrow G / H$ is the corresponding quotient homomorphism of groups. Define the sequence $S / H$ of elements of $G / H$ by setting $S / H=(q(s) ; s \in S)$. If $s \neq t$ but $q(s)=q(t)$, we represent $q(s)$ and $q(t)$ as distinct members of $S / H$. We can now construct the Cayley graph $\operatorname{Cay}(G / H, S / H)$ provided that the sequence $S / H$ does not contain the identity of the group $G / H$, that is, provided that $S$ contains no element of $H$. In this case the quotient homomorphism $q$ naturally extends to a graph homomorphism $q: \operatorname{Cay}(G ; S) \rightarrow \operatorname{Cay}(G / H, S / H)$. Since $|S / H|=|S|$, it is easy to see that $q$ is a covering projection. Note that the quotient Cayley graph $\operatorname{Cay}(G / H, S / H)$ may have parallel edges even when $\operatorname{Cay}(G, S)$ was simple.

The above considerations can be summarised as follows.

Proposition 4.1 Let $G$ be a group and $H$ a normal subgroup of $G$. Let $S$ be a connection sequence containing no element of $H$. If $\mathrm{Cay}(G / H, S / H)$ has a nowhere-zero $k$-flow, then so does $\operatorname{Cay}(G, S)$.

Now we are in the position to prove our main result.

Theorem 4.2 Let $G$ be a finite group in which a Sylow 2-subgroup constitutes a direct factor of $G$. Then every Cayley graph $\operatorname{Cay}(G, S)$ of valency at least four has a nowhere-zero 3-flow. 
Proof Let $H$ be a Sylow 2-subgroup of $G$, and assume that $H$ is a direct factor of $G$. Then there is a normal subgroup $K \leq G$ of odd order such that $H K=G$ and $H \cap K=1$. If $H=1$, then $G$ itself has odd order and Cay $(G, S)$ has even valency. In this case $\operatorname{Cay}(G, S)$ has a nowhere-zero 2-flow, and there is nothing to prove. Thus we can assume that $H \neq 1$ (implying that $G$ has even order), and that $\operatorname{Cay}(G, S)$ has odd valency (implying that $S$ contains an involution). We now proceed by induction on the order of $H$.

If $|H|=2$, then the generator $h$ of $H$ is the only involution of $G$; in particular, $h \in Z(G)$. By our assumption, $S$ contains an involution. So $h$ belongs to $S$, and the result follows from Theorem 3.3.

For the induction step assume that $|H|>2$. Since $H$ is a direct factor of $G$, the centre of $H$ is contained in the centre of $G$, and hence $H$ contains a central involution $c$ of $G$. If $c$ belongs to the connection sequence, then $\operatorname{Cay}(G, S)$ has a nowhere-zero 3-flow on the basis of Theorem 3.3. If not, then $\langle c\rangle=C$ is a normal subgroup of $G$ not intersecting $S$. We now consider the Cayley graph $\operatorname{Cay}(G / C, S / C)$. Clearly, $H / C$ is a Sylow 2-subgroup of $G / C$ and also is a direct factor of $G / C$. By the induction hypothesis, $\operatorname{Cay}(G / C, S / C)$ has a nowhere-zero 3-flow, and by Proposition 4.1 the same is true for $\operatorname{Cay}(G, S)$.

Theorem 4.2 has the following important corollary.

Theorem 4.3 Every Cayley graph of valency at least four on a nilpotent group has a nowhere-zero 3-flow.

Proof The result immediately follows from Theorem 4.2 and from the fact that every finite nilpotent group is the direct product of its Sylow subgroups (see [12, Theorem 5.31]).

Acknowledgement This research was partially supported by APVV-0111-07 and by VEGA grant 1/3022/06.

\section{References}

1. Bondy, J.A., Murty, U.S.R.: Graph Theory with Applications. Elsevier, New York (1976)

2. DeVos, M., Xu, R., Yu, G.: Nowhere-zero $\mathbb{Z}_{3}$-flows through $\mathbb{Z}_{3}$-connectivity. Discrete Math. 306, 26-30 (2006)

3. Diestel, R.: Graph Theory, 3rd edn. Springer, Heidelberg (2005)

4. Gross, J.L., Tucker, T.W.: Topological Graph Theory. Wiley, New York (1987)

5. Grötzsch, H.: Ein Dreifarbensatz für dreikreisfreie Netze auf der Kugel, Wiss. Zeitschrift d. M.-L.Universität Halle-Wittenberg. Math.-Naturwiss. Reihe 8, 109-120 (1958/1959)

6. Imrich, W., Škrekovski, R.: A theorem on integer flows on Cartesian products of graphs. J. Graph. Theory 43, 93-98 (2003)

7. Jaeger, F.: Nowhere-zero flow problems. In: Beineke, L.W., Wilson, R.J. (eds.) Selected Topics in Graph Theory, vol. 3, pp. 71-95. Academic Press, London (1988)

8. Kochol, M.: An equivalent form of the 3-flow conjecture. J. Combin. Theory Ser. B 83, 258-261 (2001)

9. Kochol, M.: Equivalence between Hamiltonicity and flow conjectures, and the sublinear defect property. Discrete Math. 254, 221-230 (2002)

10. Lai, H.-J.: Nowhere-zero 3-flows in locally connected graphs. J. Graph Theory 42, 211-219 (2003) 
11. Potočnik, P., Škoviera, M., Škrekovski, R.: Nowhere-zero 3-flows in Abelian Cayley Graphs. Discrete Math. 297, 119-127 (2005)

12. Rotman, J.J.: An Introduction to the Theory of Groups, 4th edn. Springer, New York (1999)

13. Shu, J., Zhang, C.-Q.: Nowhere-zero 3-flows in products of graphs. J. Graph Theory 50, 79-89 (2005)

14. Steinberg, R., Younger, D.: Grötzsch's theorem for the projective plane. Ars Combin. 28, 15-31 (1989)

15. Sudakov, B.: Nowhere-zero flows in random graphs. J. Combin. Theory Ser. B 81, 209-223 (2001)

16. Tutte, W.T.: A contribution to the theory of chromatic polynomials. J. Can. Math. Soc. 6, 80-91 (1954)

17. Tutte, W.T.: On the algebraic theory of graph colorings. J. Combin. Theory 1, 15-50 (1966)

18. Xu, R., Zhang, C.-Q.: Nowhere-zero 3-flows in squares of graphs. Electronic J. Combin. 10, R5 (2003)

19. Zhang, C.-Q.: Integer Flows and Cycle Covers of Graphs. Dekker, New York (1997)

20. Zhang, C.-Q.: Circular flows of nearly Eulerian graphs and vertex-splitting. J. Graph Theory 40, 147151 (2002) 